AperTO - Archivio Istituzionale Open Access dell'Università di Torino

\title{
Lipid nanoparticles for intranasal administration: application to nose-to-brain delivery
}

\section{This is the author's manuscript}

Original Citation:

\section{Availability:}

This version is available http://hdl.handle.net/2318/1662297

since 2018-05-12T22:59:49Z

Published version:

DOI:10.1080/17425247.2018.1429401

Terms of use:

Open Access

Anyone can freely access the full text of works made available as "Open Access". Works made available under a Creative Commons license can be used according to the terms and conditions of said license. Use of all other works requires consent of the right holder (author or publisher) if not exempted from copyright protection by the applicable law. 
This is the author's final version of the contribution published as:

Lipid nanoparticles for intranasal administration:application to nose-to-brain delivery

Luigi Battaglia, Pier Paolo Panciani, Elisabetta Muntoni, Maria Teresa Capucchio, Elena Biasibetti, Pasquale De Bonis, Silvia Mioletti, Marco Fontanella \& Shankar Swaminathan

EXPERT OPINION ON DRUG DELIVERY, 2018

VOL. 15 , NO. 4, 369-378

The publisher's version is available at:

https://www.tandfonline.com/

When citing, please refer to the published version.

\section{Link to this full text:}

http://hdl.handle.net/

https://www.tandfonline.com/doi/full/10.1080/17425247.2018. 


\title{
Lipid nanoparticles for intranasal administration: application to nose-to-brain delivery
}

\author{
Battaglia L1, Panciani PP2, Muntoni E1, Capucchio MT³, Biasibetti E3, \\ De Bonis $\mathbf{P}^{4}$, Mioletti $\mathbf{S}^{3}$, Fontanella $\mathbf{M}^{5}$, Swaminathan $\mathbf{S}^{6}$
}

1) University of Torino, Department of Drug Science and Technology, Torino, IT

2) Spedali Civili di Brescia, Neurosurgery, Brescia, IT

3) University of Torino, Department of Veterinary Sciences, Torino, IT

4) University of Ferrara, Neurosurgery, Ferrara, IT

5) University of Brescia, Neurosurgery, Brescia, IT

6) Perrigo Company Minneapolis, Product Development, Minneapolis, MN, USA

\section{Keywards}

Neurodegenerative diseases; Intranasal delivery; Lipid nanoparticles; Drug delivery 


\title{
Lipid nanoparticles for intranasal administration:
}

\section{application to nose-to-brain delivery}

\author{
Keywords \\ Neurodegenerative diseases; Intranasal delivery; Lipid nanoparticles; Drug delivery
}

\section{Introduction}

\subsection{The Blood Brain Barrier (BBB)}

Recent research efforts have been aimed toward targeting drugs to the brain, since $98 \%$ of current marketed pharmaceuticals are ineffective in treating cerebral diseases, mainly because of the presence of BBB. Unlike peripheral capillaries, those of the brain present no fenestrae, and fewer pinocytic vesicles, in addition to the presence of tight junctions, also known as zonula occludens, closely regulating the movement of molecules through the paracellular pathway [1]. Additionally, on the luminal side of endothelial cells, the Pglycoprotein (P-gp) system, an ATP-dependent drug transport protein, can hinder the accumulation of many molecules in the brain, by expelling them from endothelial cells towards the bloodstream [2]. As paracellular diffusion is very limited, the transport of molecules from the bloodstream through the BBB can only take place by transcellular pathway (high lipophilicity and molecular weight $<450 \mathrm{Da}$ ), carrier-mediated transport and receptor-mediated endocytosis.

Methods that may enhance drug delivery to the brain are thus of great interest. Overcoming of the BBB may be achieved by using colloidal systems (i.e., micelles, liposomes, nanoparticles) administered through the intravenous (iv) route. However, after iv administration, colloidal systems strongly interact with plasma proteins, through the opsonization process, and are rapidly removed from the circulation by the macrophages of the liver and the spleen. Only small and hydrophilic-surface coated colloidal particles can escape opsonization, and remain in the bloodstream for relatively prolonged time periods, enabling brain uptake: in this case, they can directly trigger the endothelial cells by inducing endocytosis, which may also be receptor-mediated through an active targeting mechanism, obtained by surface functionalization [3].

\section{iris-AperTO}


Alternative brain delivery strategies include [4]:

(a) trans-cranial drug delivery to the brain, i.e. by intracerebroventricular (ICV) injection, intra-cerebral (IC) implantation and convection-enhanced diffusion (CED). The ICV administration of glial-derived neurotrophic factor (GDNF) was recently attempted for the treatment of Parkinson's disease [5]. An example of depot formulations for IC implantation is the Gliadel Wafer (polifeprosan 20 with carmustine implant), which is indicated for patients with newly diagnosed or recurrent high-grade malignant glioma, in addition to radiation and/or surgery.

(b) BBB disruption in which BBB can be transiently disrupted by a variety of means such as intracarotid arterial infusion of hyperosmotic solutions, noxious agents including vasoactive compounds or local ultrasonic irradiation of the brain.

\subsection{Intranasal delivery}

Nose to brain delivery is the simpler and direct way for brain targeting, which avoids bloodstream clearance and invasive methods. It works due to the unique connection provided by the olfactory and trigeminal nerves between the brain and external environments: following intranasal administration, drugs are exposed to the nasal mucosa, which is innervated by olfactory and trigeminal nerves [6,7]. Thus, the major routes of intranasal delivery are the olfactory (olfactory epithelium) and the trigeminal nerve pathways (respiratory epithelium).

The nasal cavity can be divided into four parts: nasal vestibule, nasal atrium, respiratory epithelium and olfactory epithelium. The olfactory epithelium is formed by various cell types, and by the underlying lamina propria, which contains blood vessels, axons, glands and connective tissue; the respiratory region, instead consists of ciliated epithelial cells and mucous secreting goblet cells.

Mucus or nasal secretion is present in both the two epithelium layers, in order to favour mucociliary clearance (MCC). The cilia beat with a frequency of 1000 strokes per minute: hence the mucus moves only from the anterior to the posterior part of the nasal cavity, until the back of the throat, with a speed of $5 \mathrm{~mm}$ per minute. MCC regulates residence time of the drug in nasal mucosa [6].

The olfactory epithelia are the most likely site for direct nose to brain delivery: it chiefly consists of olfactory neural cells, the sustentacular (also known as supporting) cells and the

\section{iris-AperTO}


basal cells. Basal cells are progenitor cells (of supporting cells), that also provide mechanical support via anchorage to other cells. The olfactory neural cells or the axons are unmyelinated and interspaced between the supporting cells. They originate at the olfactory bulb in the brain and terminate at the apical surface of the olfactory epithelium. The olfactory knob protrudes out from and above the apical surface of the olfactory epithelium. Approximately 10-23 cilia project from the basal bodies of the knob, each of length up to $200 \mu \mathrm{m}$. The cilia are nonmotile in the olfactory region in contrast to respiratory region. The average diameter of olfactory axons in human is in the range 100-700 nm. Therefore, theoretically transcellular transport of nanoparticles is possible. In the olfactory region, neurons are interspersed among supporting cells and basal cells to form the olfactory epithelium [6]. Moreover, due to the direct contact with toxins in the external environment, olfactory receptor neurons regenerate every 3-4 weeks from basal cells residing in the olfactory epithelium. As a result, proteins characteristic of the BBB (tight junction proteins, efflux transporters), which are present in the nasal passages, may not be fully functional during the maturation of neurons. The nasal barrier to the CNS could be considered "leaky"because of neurons constant turnover [8].

Another important pathway for nose to brain delivery is the trigeminal nerve, which innervates the respiratory, but also the olfactory epithelium, and enters the CNS in the pons. The ophthalmic (ethmoidal nerves) and maxillary (nasal branches) divisions of the trigeminal nerve enter the brain from the respiratory epithelium through anterior lacerated foramen near the pons and through the cribriform plate near olfactory bulb. This unique feature creates entry points into both caudal and rostral brain areas. Because the entry through the cribriform plate is common to the olfactory and the trigeminal nerves, it is difficult to distinguish whether intranasally administered drugs reach the olfactory bulb and other rostral brain areas via the olfactory or trigeminal pathways or if both pathways are involved [8].

Transfer of substances from the nose into the brain can occur by slow intra-axonal transport (intra-neuronal), or by faster transfer along the perineural space surrounding the nerve cells into the cerebrospinal fluid (CSF), and/or into the interstitial fluid of the brain (extraneuronal or paracellular transport] [9]. Some substances may be transported by the intracellular pathway within neurons of the olfactory epithelium following adsorptive, receptor-mediated, or non-specific fluid phase endocytosis. If they cross the olfactory epithelium, they can reach the olfactory bulb by intraneuronal transport. Alternatively, 
intracellular pathways across the respiratory epithelium potentially include endocytosis into trigeminal nerve processes resulting in intracellular transport to the brainstem. This pathway involves the axonal transport of lipid soluble molecules, and requires a long time to reach different brain regions $[10,11]$. However, intracellular mechanisms, while important for certain therapeutics, are not likely to be the predominant mode of transport into the CNS. The vast majority of published intranasal studies demonstrate rapid delivery, with high CNS concentrations and effects observed almost immediately after or within an hour of intranasal administration, consistent with rapid extracellular mechanisms of transport: substances may cross either the olfactory or respiratory epithelia by paracellular transport, reaching the lamina propria and then the cranial compartment or the general systemic circulation [10]. This route is associated with tight junctions, intracellular space, gaps or pores present between the epithelial cells, and constitutes an important pathway for the absorption of small (less than $1000 \mathrm{Da}$ ), polar or hydrophilic substances and peptides, but it is less efficient, with respect to intra-axonal pathway; however this mechanism is quite fast (within few minutes after administration) [6].

It should be poited out that several enzymes are present in the nasal cavity such as cytochrome P-450 enzyme isoforms, carboxylesterases and glutathione S-transferases which may play a role in metabolism of drugs intranasally. The drug delivery to the brain is also limited by ATP binding cassette (ABC) drug efflux transporter system present in nose [6,7]. Furthermore, since the blood supply to the respiratory epithelium is relatively high compared to olfactory epithelium, it is an ideal site for systemic absorption of nasally applied drugs, in order to bypass first pass effect- a common drawback of oral drug delivery [12]. An overview of different pathways associated with intranasal delivery is presented in Figure 1.

\section{Lipid nanoparticles for intranasal delivery}

Nanoparticles can improve nose-to-brain drug delivery, since they are able to protect the encapsulated drug from biological and/or chemical degradation, and from extracellular transport by P-gp efflux proteins [6]. Moreover, retention of bioadhesive nanoparticles on the mucosal surface of nasal cavity, as well as their ability of transiently opening of the tight junctions of the mucosal epithelium (because of surfactants used in the formulation) should contribute to enhanced brain drug delivery [13]. The transmucosal transport of nanoparticles

\section{iris-AperTO}


from nose to brain may take place by through olfactory neurons via various endocytic pathways of sustentacular or neuronal cells. Confocal microscopy revealed that fluorescent labelled polystyrene nanoparticles of diameter in the range of 20-200 nm were involved with clathrin-coated pits whereas nanoparticles of size in the range of 200-1000 nm were found to be involved with caveole-mediated endocytosis [14]. Molecules can also be transferred from endothelial cells to olfactory neurons via endocytosis or pinocytosis and move along the axon up to the olfactory bulb. However, since axon diameter is about $100-700 \mathrm{~nm}$, the transport of colloidal systems within this size range might be highly limited [15].

One of the key considerations for development of nasal dosage forms is their safety and toxicological assessment of the matrix. The extended contact of formulations with nasal mucosa may lead to irritation, tissue damage, epithelial/sub epithelial toxicity or ciliotoxicity, and may result in environment suitable for microbial growth. In addition, intranasal drug formulation should not damage of the primary olfactory nerves and the sense of smell [16]. This is why, despite some experimental approaches have been recently attempted with noseto-brain delivery for glioma treatment [17], this administration route is problematic for cytotoxic drugs, that can cause local damage in the nose cavity after administration. Among colloidal carriers, vesicular systems (liposomes, niosomes), nanoemulsions, lipid, and polymeric nanoparticles are the most promising for nasal drug delivery, especially with regard to their biocompatibility. In particular, lipid nanoparticles (LN) are particles in the 50$1000 \mathrm{~nm}$ range, wherein the matrix is made of biocompatible solid lipids or mixtures of solid and liquid lipids. So far, when compared to other colloidal systems, LN have been described as superior carriers, with various advantages [18].

Solid lipid nanoparticles (SLN) are considered to be among the most effective lipid-based colloidal vehicles. They are constituted by a solid lipid matrix (triglycerides, waxes, sterols, fatty acids) surrounded by a layer of surfactants in an aqueous dispersion. Nanostructured lipid carriers (NLCs) are LN characterized by a solid lipid core consisting of a mixture of solid and liquid lipids: NLC allow to increase drug payload compared to SLN, reducing drug expulsion and burst release phenomena. To overcome the limited loading capacity of hydrophilic drugs by LN, lipid-drug conjugates (LDC) nanoparticles have been developed, by synthesis of an insoluble drug-lipid conjugate bulk, that is then processed in the same way as SLN [19].

\section{iris-AperTO}


LN production can be easily scaled up, and can be considered as physico-chemically stable formulations. However, some stability problems can occur and can be an obstacle during the scale-up process. Stability of LN can be increased by freeze-drying or spray-drying of the suspension: in the first case the employment of cryoprotectants can help in avoiding particle aggregation. Moreover, given that intranasal delivery requires sterile formulations, it should be noticed that generally LN are stable to steam sterilization; if not gamma-irradiation can employed, even if there is the risk of unsaturated lipid peroxidation. Sterile filtration can be applied only to ultrasmall LN, owing to $220 \mathrm{~nm}$ size of sterylizing filters [19].

Owing to their biocompatibility, stability, ease of surface functionalization and scale-up, LN have been recently employed for intranasal delivery for different applications. Intranasal provides several advantages over other routes of drug administrations, including fast onset of action, avoidance of the intestinal and hepatic presystemic disposition, reduction of systemic exposure and side effects, direct delivery and targeting to the brain and CSF, ease of administration and better patient compliance. Some of the limitations associated with this route are mucociliary clearance of nasally applied drugs, low permeability for hydrophilic drugs, enzymatic degradation by nasal cytochrome P450/peptidases/proteases, CNS delivery efficiency limited to potent drugs or small volumes (25200 microliters) $[4,12]$.

\subsection{Experimental models employed and evalutation methods for intranasal LN}

The experimental model employed is very important, both for the scientific goal reached, and for the assessment of the stage of development of the drug delivery system. In fact, despite many studies involve animal models, it should be considered that the anatomical differences between animal models and human are distinct. Rodents are obligatory nasal breathers, while primates are oro-nasal breathers. The nasal passage in rat is more complex than in humans, and has a larger surface-to-volume ratio. Nasal cavities in mice, rat and human present a volume of $0.032,0.26$, and $25 \mathrm{~cm}^{3}$, respectively [20].

According to the literature analyzed, the following experimental models are currently employed to study nose-to-brain delivery by LN:

- Ex-vivo diffusion study: a fresh nasal mucosa excised from animals (goat, sheep, swine) is mounted between the receptor and donor compartment of a Franz cell: diffusion through the mucosa and histology of the tissue can provide efficacy and toxicity data

iris-AperTO 
- In vivo biodistribution and pharmacokinetics after intranasal administration of 99mTc labelled LN in slightly anestethized animals. Biodistribution can be followed through gamma scintigraphy imaging and radiometric data after sacrificing the animals

- Case specific pharmacodynamic in vivo tests in animal models

Moreover, some more specific techniques can be employed to assess the mucociliary clearance of drugs and formulations, especially as a screening method to study their potential toxicity [21]:

- In vivo measurement of mucociliary clearance: saccharin test, dye method, radiographic method, radiosotopic method

- In vitro measurement of diary beat frequency on ciliated epithelium

The most important one is nose-to-brain delivery, even if some examples concerning local and systemic delivery are present in literature (Table 1).

\subsection{LN for local delivery and for first pass metabolism avoidance}

Propolis-a natural multifunctional material-shows therapeutic properties which is solid at room temperature, and composed mainly of resin and waxes, and enriched with flavonoids with therapeutic properties. In a recent study, propolis was proposed as lipid material to prepare SLN for topical intranasal therapy. Ex vivo studies on swine nasal mucosa demonstrated that the SLN based on propolis can be used as a release system for diclofenac and flavonoids for the local treatment of diseases of the nasal cavity [22].

Particular attention should be paid to the fact that intranasal administration allows to overcome first pass metabolism, which is a drawback of oral route. Carvedilol is a nonselective beta-adrenergic antagonist used in the treatment of hypertension and angina pectoris: although it is well absorbed in the gastrointestinal tract, it is extensively metabolized in the liver after oral administration, leading to decreased bioavailability of about $25 \%$. Pharmacokinetic studies of intranasally administered carvedilol loaded SLN in rabbits showed enhancement of bioavailability compared to oral administration. Fluorescently labelled SLN allowed to assess, through confocal microscopy, the penetration up to the deep layers in sheep nasal mucosa, while histopathological evaluation excluded toxic local effects (ex vivo studies) [23].

\subsection{LN for nose-to-brain delivery}


All neurodegenerative disorders, such as Parkinson's disease (PD), Alzheimer's disease (AD), Huntington's disease (HD) and Amyotrophic Lateral Sclerosis (ALS), that are treated through chronic drug therapy, are suitable for nose-to-brain approach, with the limitation of potent drugs, that can be administered in small volumes. Furthermore, alteration of the microenvironment composition for neurons and glia is regularly observed in several neurological diseases like stroke, traumatic brain injury, and normal pressure hydrocephalus, that potentially can be treated through a nose-to-brain approach [11]. Potential advantages for intranasal treatment in brain diseases were summarized in Figure 2.

The drugs reported for nose-to-brain delivery in LN are currently used in pharmacological therapy or with potentiality to be employed: some of them easily cross the BBB, but undergo side effects or fast metabolism following oral administration, while others can't be used in current therapy because of poor brain availability; in both cases, intranasal delivery can act as an important tool to improve therapeutic efficacy and patient compliance. To this aim, it should be pointed out that the parameter allows to estimate the brain targeting efficiency: a formulation or compound with a brain-blood ratio comprised between 0.3-0.5 has sufficient access to the CNS, if greater than 1.0 it freely crosses the BBB, whereas if smaller than 0.1 it may be unable to enter the CNS [24].

\subsubsection{Major neurodegenerative diseases}

\section{PD treatment}

For early PD (mild motor disability) monotherapy with monoamine oxidase (MAO-B) inhibitors can be the first treatment option; in advanced PD (mild to moderate motor disability) treatment can begin with dopamine (DA) agonists along with MAO-B inhibitors; complex PD (moderate to severe motor disability with cognitive impairment) can be treated with a combination therapy, including catechol-0-methyl transferase (COMT) inhibitors along with DA precursor (levodopa). Levodopa is administered orally and crosses BBB owing to carrier transport; other chemotherapeutics partially cross BBB, but can take advantage of nose-to-brain delivery [13].

The bioavailability of ropinirole hydrochloride (DA agonist) after oral administration is 50\% due to extensive hepatic first pass metabolism. Preparation of surface-modified SLN for the intranasal delivery of ropinirole hydrochloride has been reported; stearylamine-induced

\section{iris-AperTO}


cationic charge was engineered on SLN surface, which contributed to the enhanced entrapment efficiency and sustained drug release. The nasal toxicity studies on sheep nasal mucosa revealed the non-irritant nature of the SLN formulation. In vivo anti-tremor activity of SLN formulation in albino mice model suggested that the prepared nasal SLN formulation was superior compared to a marketed tablet formulation. The authors concluded that the SLN formulation aids in reducing the dose and dosing frequency, thereby maximizing the therapeutic index of the selected drug candidate [25].

\section{AD treatment}

Currently approved drugs for treating the cognitive impairments in AD are based on neurotransmitter or enzyme replacement/modulation. Acetylcholinesterase inhibitors are the main class of drugs used in $\mathrm{AD}$. Another approach to the treatment of $\mathrm{AD}$ is to block glutamatergic neurotransmission: memantine is a non-competitive NMDA-receptor antagonist. These treatment strategies provide only moderate benefit and thus there is an apparent need for improved therapies: in particular, since oxidative stress and $\beta$-Amiloid are considered as a major etio-pathological factor in the initiation and promotion of neurodegeneration in $\mathrm{AD}$, antioxidants, as well as peptides and nuclear factors (such as NFkB), GSK3, peroxisome proliferator-activated receptor-g9, are proposed for experimental treatment of AD. All these therapies would benefit from nose-to-brain delivery in order to increase brain accumulation [12].

Rivastigmine, an acetylcholinesterase inhibitor, was loaded in SLN, in order to avoid its extensive first-pass metabolism (resulting in reduced absolute bioavailability of only 36\%), and its important side effects after oral administration. Rivastigmine loaded SLN, being lipidic in nature, showed higher drug diffusion in comparison to drug solution having crystalline form of drug. SLN did not show any nasociliary damage and/or cell necrosis, in ex vivo studies on sheep nasal mucosa, indicating their safety for nasal administration [26]. Also, antioxidants were loaded in SLN for potential AD treatment, with the aim of improving their brain biodistribution. In particular, astaxanthin loaded SLN showed a strong neuroprotective effect against oxidative stress in neuronal cell lines. The direct nose-to-brain delivery of the $99 \mathrm{mTc}$ labelled SLN in Wistar rats caused an increased brain uptake, which was evident by gamma scintigraphy imaging and radiometric data after sacrificing the animals [27]. Tarenflurbil is a non steroidal anti-inflammatory drug, that showed potentiality also in the treatment of AD, 
but poor BBB permeation led to its failure in phase III trials. PLGA nanoparticles (NP) and SLN were evaluated for their efficacy in nose-to-brain delivery of tarenflurbil. Desirable brain biodistribution profiles were showed by tarenflurbil loaded NP and SLN in Sprague Dawley rats, and overall pharmacokinetics of tarenflurbil was improved through nanoparticles, and intranasal route was proved to be advantageous over iv and oral route of administration; NP showed comparable, but slightly better results compared to SLN, even if this aspect was not discussed by the authors [28].

Chitosan coated cationic SLN were prepared and complexed with cell penetrating peptides (CPP) and siRNA aimed to potential Alzheimer's disease treatment. The 29-amino-acid peptide derived from the rabiesvirus glycoprotein (RVG) was selected as CPP, since it binds to acetylcholine receptors, which are highly expressed in the neuronal cells. The major $\beta$ secretase responsible for amyloid- $\beta$ generation in the brain is BACE1. BACE1 siRNA specifically influence the $\beta$-cleavage of amyloid precursor proteins and is a potential therapeutic approach for treating Alzheimer's disease. The encapsulation of the complex in SLN was obtained via chitosan coating, which modified the Zeta Potential and the mucoadhesiveness of the system, as well as the permeation ability of the siRNA through the Caco-2 epithelial cells, enhancing the intracellular transport [29].

\section{HD treatment}

HD is an autosomal dominant, fully penetrant, progressive, and fatal neurodegenerative disease characterized by progressively worsening chorea, cognitive and psychiatric disturbance. Oxidative injury, transcriptional dysregulation, glutamate exitotoxicity are present, and apoptotic huntingtin is expressed more intensely in neurons than in glial cells. Currently there is no approved pharmacological therapy for HD, but experimental therapy is proposed for its symptoms. It has been reported that some antioxidants, like rosmarinic acid, protect neurons from oxidative stress-induced disease condition.

In animal HD rat models, treatment with rosmarinic acid loaded SLN by intranasal route is able to significantly attenuate 3NP-induced deficits in body weight, beam walk, locomotor and motor coordination, and are also able to significantly attenuate 3NP-induced striatal oxidative stress. Intranasal delivery also prevents unwanted distribution and metabolism in other parts of body [30].

\section{iris-AperTO}




\section{ALS treatment}

Dimethyl fumarate has been demonstrated useful in relapsing remitting ALS treatment (Tecfidera ${ }^{\circledR}$ ). Nevertheless, Tecfidera ${ }^{\circledR}$ capsules induce flushing, gastro-intestinal events and other more serious drawbacks. Tristearin SLN, simple, coated with polysorbate 80, and cationized with dimethyldioctadecylammonium chloride were compared as vehicles for intranasal delivery. An in vitro study performed through a model of mouse brain microvascular endothelial cells indicated an higher permeability from cationic SLN. Biodistribution of polysorbate 80 treated SLN was studied by fluorescent imaging after intraperitoneal or intranasal administration in mice: polysorbate 80 treated SLN were able to reach the brain both after intraperitoneal and intranasal route, even by intraperitoneal route they prevalently accumulated in liver and spleen [31].

\subsubsection{Other CNS diseases}

Anti-psychotic, antidepressant, sedative and anti-epileptic drugs are suitable candidates for LN mediated nose-to-brain delivery. The pharmacodynamic and pharmacokinetic studies performed after intranasal administration of risperidone loaded SLN in mice clearly showed that SLN have proved effective for brain targeting. Comparative biodistribution data, obtained by using $99 \mathrm{mTc}$ labelling and gamma scintigraphy in Balb/C mice, indicate that higher drug concentration in the brain was achieved by intranasal delivery of risperidone loaded SLN, as compared with free risperidone i.v. and risperidone loaded SLN i.v. The paw test was performed for pharmacodynamic activity: the increase in hindlimb retraction time (HRT) is associated with the antipsychotic potential, whereas the increase in forelimb retraction time (FRT) was associated with the potential to induce extra-pyramidal side effects (EPS). In mice there was no significant difference found in FRT among treated and controls. However, a significant rise in HRT was observed for intranasally administered SLN formulations when compared with free risperidone. Since risperidone is an atypical antipsychotic agent, which is expected to affect the HRT, but not the FRT, increase in HRT can be directly correlated to increased brain levels of the drug [32]. Agomelatine is a novel antidepressant drug suffering from an extensive first-pass metabolism leading to a diminished absolute bioavailability. Pharmacokinetics and biostribution studies, performed in rats, demonstrated that intranasal delivery of agomelatine loaded SLN enhanced its absolute bioavailability, and increased its brain delivery [33]. A longer nasal rentention time can be obtained through entrapment of 
SLN suspension in a mucoadhesive gel (chitosan, carboxymethylcellulose, hydroxypropylmethylcellulose, alginate), resulting in further improvement of brain uptake through intranasal administration [34]. Also alprazolam loaded SLN can be rapidly transferred to the brain via the intranasal delivery, as demonstrated by an experimental study employing 99mTc labelling and gamma scintigraphy in animal models. Enhanced rate and extent of transport of alprazolam loaded SLN, as compared to alprazolam solution, demonstrated in New Zealand rabbits, may help in reducing the dose and dosing frequency and may increase the therapeutic index as well as the clinical acceptability for ambulatory patients [35]. Another experimental study showed, through biodistribution studies, that intranasal administration of valproic acid loaded NLC in Wistar rats is a suitable method to increase drug brain-blood ratio. Moreover, in maximal electroshock (MES) seizure test performed in animal models, intranasally administered valproic acid loaded SLN provided the same protective effect as sodium valproate solution after i.p. administration, with a dose of about 37 times less [36].

Cerebrovascular disorders and age-related memory impairment can be treated by nose-tobrain delivery, and vinpocetine is an alkaloid with cerebral blood-flow enhancing and neuroprotective activity, which is employed, especially in Eastern Europe for this therapeutic aim. Vinpocetine loaded SLN and entrapped in ten different bioadhesive nasal gels. Ex-vivo bioadhesive strength, histopathological studies and permeation studies were performed to evaluate the prepared obtained bioadhesive nasal gels. Moreover, tissue distribution and an in vivo pharmacokinetic study were carried out. The drug targeting index was $380.46 \%$ and the nose-to-brain direct transport percentage was $73.71 \%$ suggesting a high targeting efficiency [37].

Ondansetron is a serotonin 5-hydroxy tryptamine subtype 3 receptor antagonist used in the management of chemotherapy induced postoperative nausea and vomiting. Its absolute bioavailability is about $60 \%$ due to its first pass metabolism. Ondansetron was loaded in SLN, and biodistribution was performed after 99mTclabelling in New Zealand rabbits: the radioimaging on rabbits clearly demonstrated the effectiveness of intranasal SLN delivery, while histological studies on isolated sheep nasal mucosa demonstrated the absence of toxicity [38]. Antibiotic and antiviral therapy can benefit of nose-to-brain delivery, too. Streptomycin sulfate, an aminoglycoside, is a bactericidal antibiotic active against both gram-negative and gram-positive organisms, and Mycobacterium. As antitubercular drug (ATD) it is especially 
suggested for patients with hepatitis induced by other hepatotoxic oral ATD, and for tuberculous meningitis. Streptomycin sulphate, however, has low permeability of the BBB, and suffers from serious risk of nephrotoxic reactions and irreversible ototoxicity after i.v. administration at high doses. In a recent experimental work streptomycin was entrapped in SLN and formulation was radiolabelled with 99mTc. Encouraging results obtained from biodistribution in Balb/C mice through gamma scintigraphy after intranasal administration allow to predict improvement of patient compliance at lower drug dose, with reduced side effects and increase of efficacy against tubercular meningitis [39]. The CNS is reported to be the most important HIV reservoir site, while the non nucleoside reverse transcriptase inhibitors efavirenz, used for the treatment of HIV infections, are reported to have low bioavailability, pertaining to high first-pass metabolism, high protein binding, and enzymatic metabolism, as well as low permeability across the BBB. SLN were formulated for efavirenz intransal delivery. Promising in vivo pharmacokinetic studies revealed increased concentration of the drug in brain, when administered through intranasal route indicating its potential for an attempt towards complete eradication of HIV in infected patients [40].

\subsection{Design of intransal formulations and marketed products}

Nasal preparations are formulated to be similar to nasal secretion with regards to toxicity, $\mathrm{pH}$, viscosity so that normal ciliary action is maintained. Thus, aqueous nasal formulations are isotonic and slightly buffered to maintain a $\mathrm{pH}$ of 5.5 to 6.5. In addition, antimicrobial preservatives, similar to those employed for ophthalmic formulations, and appropriate stabilizers, if required, are included in the formulation [41]. Nasal formulations are currently ruled by the FDA [42].

In the last decades the intranasal route has been mainly exploited for systemic delivery of small molecular weight drugs, especially where a rapid onset of action is required. Examples of such marketed nasal product are drugs for treatment of migraine e.g. zolmatriptan (Zomig $($ ), sumatriptan (Imigran $®$ ) and butorphanol tartrate (Stadol NS), treatment of severe pain e.g. fentanyl (PecFent $($; Instanyl $囚)$, for smoking cessation (Nicorette $®$ ) and for treatment of menopausal symptoms (Aerodiolß). None of these products contains nasal absorption enhancers due to the fact that these molecules in general are sufficiently lipophilic to enable therapeutic levels of the drug to reach the systemic circulation [43].

\section{iris-AperTO}


Moreover a range of nasal peptide products are presently on the market such as calcitonin (Miacalcin $®$, Novartis; Fortical $®$, Unigene), desmopressin (Desmospray $®$, Ferring), buserelin (Suprecur $®$, Sanofi-Aventis), nafarelin (Synarel $®$, Pharmacia) and oxytocin (Syntocinon $®$, Unites Pharmacies UK), due to the low systemic levels needed to obtain a therapeutic effect, regardless of their poor bioavailability [44].

However, many other small molecular weight drugs would be in need of a nasal promoter in order to be transported across the nasal membrane in sufficient quantity for therapeutic use. Major problem with nose-to-brain delivery is first of all the difficulty in reaching the olfactory region in the nose, secondly, at least for peptides and proteins, to increase the transport across the olfactory membrane. Hence, what is needed is a nasal device that can deliver the formulation to the olfactory region, and a delivery system including a nasal absorption enhancer and a bioadhesive material that can provide an increased penetration of the drug into the CNS.

But the poor nasal tolerability of many potential absorption enhancers is troublesome. Presently, a small group of nasal absorption enhancer systems is being commercially developed as nasal delivery systems for delivery of small molecular weight drugs and specific peptides and proteins, with an acceptable toxicity profiles and balance between safety and efficiency. Examples include CPE-215® (cyclopenta decalactone (azone)) developed by CPEX Pharma, Intravail@ (alkylsaccharides) developed by Aegis Therapeutics, ChiSysTM (chitosan) and PecSysTM (low methylated pectin) in development by Archimedes Pharma and CriticalSorbTM (polyglycol mono- and diesters of 12-hydroxystearate (70\%), polyethylene glycol (30\%)) developed by Critical Pharmaceuticals [44].

Currently, no formulation including LN for intranasal delivery is on the market, since it is a relatively recent approach. However, from literature examples it can be assessed that LN work as a bioadhesive vehicles and they exhibit also properties of permeation enhancers. In fact, the percent dose of intranasally administered drugs reaching the CNS is small, generally below 1\%, while delivery through intransal LN, employed in experimental models, allows an higher permeation into the CNS. Moreover the versatility and ease of functionalization of LN allows the association with bioadhesive polymers (i.e. chitosan) and/or other permeation enhancers that can be loaded in the lipid matrix or linked to the surface of nanoparticles, obtaining a synergic effect. 


\section{Expert opinion}

Drug development in the area of brain diseases has been fraught with challenges for decades. If the unfortunate AD clinical trial performances from 2016 (solanezumab) and early 2017 (verubecestat) are to be considered as a yard stick, it is clear that new targets and treatments for such diseases are going to be hard to come by; coupled with the recalcitrant brain barriers, finding an effective therapy is very challenging. The strategically unique structure of BBBalthough vital for keeping the harmful entities out-is also a major roadblock for delivering drug substances to the brain for treating brain diseases. Several alternative invasive approaches such as transcranial drug delivery, disruption of BBB have been explored, with limited success and several challenges. Intranasal delivery-a non-invasive methodologybypasses the systemic circulation, and via the intra- and extra- neuronal pathways provides direct access to the brain to deliver drugs.

In the past decade or so, nanotechnology based pharmaceuticals have been very successful in applications such as cancer, haematological diseases, anesthesia, retinal diseases, hepatitis B, acute radiation syndrome, diagnostics, imaging etc. Among nanoparticulate systems, LN show a better biocompatibility profile, being constituted from GRAS excipients: this is very important for intransal delivery, because of the absence of irritation, tissue damage, epithelial/sub epithelial toxicity or ciliotoxicity. Moreover, compared to other kind of nanoparticles, they are easy to scale-up, and they can be steam sterilized. They share with polymeric nanoparticles the ability to be easily functionalized and the bioadhesive properties for the nasal mucosa.

The potential application of $\mathrm{LN}$ for intransal route is wide. Brain diseases are the most important target, even if it can be also employed for drugs with a systemic target, associated to high first pass metabolism. In fact, key degenerative brain diseases, such as AD, PD, HD and ALS can benefit by treatment by this administration route, which is ideal for brain chronic diseases. Moreover, also brain diseases, not from neurodegenerative origin, can potentially be treated by this approach.

Main limitations of this approach are: the small volume administered, as well as the limited drug payload, that means only potent drugs can be employed successfully; the individual variability of nasal uptake, as well as its susceptibility to altered nasal mucosa (rhinitis, etc.). But the advantages in terms of patient compliance, fast onset of action and efficient BBB

\section{iris-AperTO}


bypassing, with consequent reduction of systemic exposure and side effects, can overcome the existing drawbacks.

Moreover, currently the pharmacological therapy is not efficient for different brain diseases, especially for neurodegenerative ones. This fact drives to find new therapeutical targets, thanks to the medicinal chemistry: the major part of newly synthesized compounds, undergoing preclinical and clinical testing for these diseases, belongs to the IV BCS class, which is characterized from low solubility and permeability, that hampers the in vivo performance of these drug candidates. Coupling a suitable lipid colloidal system with a safe and efficient administration route can overcome this limit and increase the therapeutic potential.

Brain diseases present complex pathophysiology, in addition to the practically inaccessible brain tissues, hence according to the authors, in the future a two-pronged approach utilizing new target discovery coupled with new drug delivery systems, such as lipid carriers for intransal delivery, must be adopted.

\section{Acknowledgements}

The authors thank Italian Ministry of Univeristy (MIUR) for funding (Ricerca Locale 2015).

\section{References}

1 - P. Blasi, S. Glovagnoli, A. Schoubben, et al. Solid lipid nanoparticles for targeted brain drug delivery, Adv. Drug Deliv. Rev. 59 (2007) 454-77

2 - M. Patel, E.B. Souto, K.K. Singh. Advances in brain drug targeting and delivery: limitations and challenges of solid lipid nanoparticles. Expert Opin. Drug Deliv. 10 (2013) 889-905

3 - L. Gastaldi, L. Battaglia, E. Peira, et al. Solid lipid nanoparticles as vehicles of drugs to the brain: Current state of the art. Eur. J. Pharm. Biopharm. 87 (2014) 433-44

4 - M. Shadab, M. Gulam, B. Sanjula et al. Nanoneurotherapeutics approach intended for direct nose to brain delivery. Drug Dev. Ind. Pharm. 41 (2015) 1922-34

5 - J.G. Nutt, K.J. Burchiel, C.L.Comella, et al. Randomized, double-blind trial of glial cell linederived neurotrophic factor (GDNF) in PD. Neurology 60 (2003) 69-73.

6 - A. Mistry, S. Stolnik, L. Illum. Nanoparticles for direct nose-to-brain delivery of drugs. Int. J. Pharm. 379 (2009) 146-57

\section{iris-AperTO}


7 - D. Mittal, A. Ali, S. Md, et al. Insights into direct nose to brain delivery: current status and future perspective. Drug Deliv. 21 (2014) 75-86

8 - S.V. Dhuria, L.R. Hanson,W.H. Frey. Intranasal delivery to the central nervous system: mechanisms and experimental considerations. J. Pharm. Sci. 2010 (99) 1654-1673

9 - P.G. Djupesland, J.C. Messina, R.A. Mahmoud. The nasal approach to delivering treatment for brain diseases: an anatomic, physiologic, and deliverytechnology overview, Ther. Deliv. 2014 (5) 709-733.

10 - J.J. Lochhead, R.G. Thorne. Intranasal delivery of biologics to the centralnervous system, Adv. Drug Deliv. Rev. 2012 (64) 614-628.

11 - C. Johanson, N. Johanson. Merging Transport Data for Choroid Plexus with Blood-Brain Barrier to Model CNS Homeostasis and Disease More Effectively. CNS Neurol Disord Drug Targets. 2016 (15) 1151-80

12 - S. Sood, K. Jain, and K. Gowthamarajan. Intranasal therapeutic strategies for management of Alzheimer's disease. J. Drug Target. 2014 (22) 279-94

13 - A.D. Kulkarni, Y.H. Vanjari, K.H. Sancheti, et al. Nanotechnology-mediated nose to brain drug delivery for Parkinson's disease: a mini review. J. Drug Target. 2015 (23) 775-88

14 - J. Rejman, V. Oberle, I.S. Zuhorn, et al. Size-dependent internalization of particles via the pathways of clathrin- and caveolae-mediated endocytosis. Biochem J 2004 (377) 159-69

15 - M.A. Lauzon, A. Daviaua, B. Marcos, et al. Nanoparticle-mediated growth factor delivery systems: A new way to treat Alzheimer's disease. J. Control. Release 206 (2015) 187-205

16 - N. Haffejee, J. Du Plessis, D.G. Muller, et al. Intranasal toxicity of selected absorption enhancers. Pharmazie 2001 (56) 882-8

17 - M. van Woensel, N. Wauthoz, R. Rosière, K. Amighi, V. Mathieu, F. Lefranc, S.W. van Gool and S. de Vleeschouwer. Formulations for intranasal delivery of pharmacological agents to combat brain disease: a new opportunity to tackle GBM? Cancers 2013 (5) 1020-48

18 - W. Mehnert, K. Mäder. Solid lipid nanoparticles: production, characterization and applications. Adv. Drug Deliv. Rev. 2001 (47) 165-96

19 - L. Battaglia, M. Gallarate. Lipid nanoparticles: state of the art, new preparation methods and challenges in drug delivery. Expert Opin. Drug Deliv. 2012 (9) 497-508*

20 - B.W. Jafek. Ultrastructure of human nasal mucosa. Laryngoscope 1983 (93) 1576-99

21 - C. Ruszniak, J.L. Devalia, S. Lozewicz et al. The assessment of nasal mucociliary clearance and the effect of drugs. Respiratory Medicine 1994 (88) 89-101

\section{iris-AperTO}


22 - G. Rassu, M. Cossu, R. Langasco, et al. Propolis as Lipid Bioactive Nano-Carrier for Topical Nasal Drug Delivery. Coll. Surf. B: Biointerf. 2015 (136) 908-917

23 - H.M. Aboud, M.H. El Komy, A.A. Ali, et al. Development, Optimization, and Evaluation of Carvedilol-Loaded Solid Lipid Nanoparticles for Intranasal Drug Delivery. AAPS PharmSciTech 2016 (17) 1-13

24 - A. Reichel. The role of blood-brain barrier studies in the pharmaceutical industry. Curr. Drug Metab. 2006 (7) 183-203 **

25 - C.V. Pardeshi, V.S. Belgamwar, A.R. Tekade, et al. Novel Surface Modified Polymer-Lipid Hybrid Nanoparticles as Intranasal Carriers for Ropinirole Hydrochloride: In Vitro, Ex Vivo and in Vivo Pharmacodynamic Evaluation. J. Mater. Sci. Mater. Med. 2013 (24) 2101-15.

26 - B. Shah, D. Khunt, H. Bhatt, et al. Application of Quality by Design Approach for Intranasal Delivery of Rivastigmine Loaded Solid Lipid Nanoparticles: Effect on Formulation and Characterization Parameters. Eur. J. Pharm. Sci. 2015 (78) 54-66.

27 - P. Chandra Bhatt, P. Srivastava, P. Pandey, et al. Nose to Brain Delivery of AstaxanthinLoaded Solid Lipid Nanoparticles: Fabrication, Radio Labeling, Optimization and Biological Studies. RSC Adv. 2016 (6) 10001-10

28 - E. Muntimadugu, R. Dhommati, A. Jain, et al. Intranasal Delivery of Nanoparticle Encapsulated Tarenflurbil: A Potential Brain Targeting Strategy for Alzheimer's Disease. Eur. J. Pharm. Sci. 2016 (92) 224-34

29 - G. Rassu, E. Soddu, A.M. Posadino. Nose-to-brain delivery of BACE1 siRNA loaded in solid lipid nanoparticles for Alzheimer's therapy. Colloid Surf. B: Biointerfaces 2017 (152) 296-301 30 - R. Bhatt, D. Singh, A. Prakash, et al. Development, Characterization and Nasal Delivery of Rosmarinic Acid-Loaded Solid Lipid Nanoparticles for the Effective Management of Huntington's Disease. Drug Deliv. 2015 (22) 931-9

31 - E. Esposito, R. Cortesi, M. Drechsler et al. Nanoformulations for dimethyl fumarate: Physicochemical characterization and in vitro/in vivo behavior. Eur. J. Pharm. Biopharm. 2017 (115) 285-96.

32 - S. Patel, S. Chavhan, H. Soni, et al. Brain Targeting of Risperidone-Loaded Solid Lipid Nanoparticles by Intranasal Route. J. Drug Target. 2011 (19) 468-74

33 - A.M. Fatouh, A.H. Elshafeey, A. Abdelbary. Agomelatine-based in situ gels for brain targeting via the nasal route: statistical optimization, in vitro, and in vivo evaluation. Drug Deliv. 2017 (24) 1077-85

\section{iris-AperTO}


34 - A.M. Fatouh, A.H. Elshafeey, A. Abdelbary. Intranasal agomelatine solid lipid nanoparticles to enhance brain delivery: formulation, optimization and in vivo pharmacokinetics. Drug Des Devel Ther. 2017 (11) 1815-25.

35 - A.P. Singh, S.K. Saraf, and S.A. Saraf. SLN Approach for Nose-to-Brain Delivery of Alprazolam. Drug Deliv. Transl. Res. 2012 (2) 498-507

36 - S. Eskandari, J. Varshosaz, M. Minaiyan, et al. Brain Delivery of Valproic Acid via Intranasal Administration of Nanostructured Lipid Carriers: In Vivo Pharmacodynamic Studies Using Rat Electroshock Model. Int. J. Nanomedicine 2011 (6) 363-71

37 - N.M. Morsi, D.M. Ghorab, H.A. Badie. Bioadhesive brain targeted nasal delivery of an ant ischemic drug. Pharma Research 2013 (8) 43-61

38 - A.S. Joshi, H.S. Patel, V.S. Belgamwar, et al. Solid Lipid Nanoparticles of Ondansetron HCl for Intranasal Delivery: Development, Optimization and Evaluation. J. Mater. Sci. Mater. Med. 2012 (23) 2163-75

39 - M. Kumar, V. Kakkar, A.K. Mishra, et al. Intranasal Delivery of Streptomycin Sulfate (STRS) Loaded Solid Lipid Nanoparticles to Brain and Blood. Int. J. Pharm. 2014 (461) 223-33

40 - S. Gupta, R. Kesarla, N. Chotai et al. Systematic Approach for the Formulation and Optimization of Solid Lipid Nanoparticles of Efavirenz by High Pressure Homogenization Using Design of Experiments for Brain Targeting and Enhanced Bioavailability. Biomed. Res. Int. 20175984014

41 - D.B. Troy, P. Beringer. Remington: The Science and Practice of Pharmacy. Lippincott Williams \& Wilkins Editors

42 - U.S. Department of Health and Human Services Food and Drug Administration (FDA) Center for Drug Evaluation and Research (CDER). Guidance for Industry Nasal Spray and Inhalation Solution, Suspension, and Spray Drug Products - Chemistry, Manufacturing, and Controls. Documentation. July 2002

43 - L. Illum, Nasal drug delivery: new developments and strategies, Drug Discov. Today 2002 (7) 1184-1189.

44 - L. Illum Nasal drug delivery - Recent developments and future prospects. J. Control. Rel. 2012 (161) 254-263

*=of importance: it concerns the main lipid nanoparticles described in literature 
$*^{* *}=$ of considerable importance: it concerns the industrial applicability of lipid nanoparticles for BBB overcoming 


\begin{tabular}{|c|c|c|c|c|c|c|c|}
\hline Therapeutic aim & \multicolumn{2}{|c|}{ Drug category } & Drug & LN type & Lipid employed & Assays performed & Ref. \\
\hline Local delivery & \multicolumn{2}{|c|}{ Anti-rhinitis } & $\begin{array}{l}\text { Flavonoids, } \\
\text { diclofenac }\end{array}$ & SLN & Propolis & $\begin{array}{l}\text { ex vivo permeation studies on swine nasal } \\
\text { mucosa }\end{array}$ & 22 \\
\hline $\begin{array}{l}\text { Bypassing first } \\
\text { pass metabolism }\end{array}$ & \multicolumn{2}{|c|}{ Anti-hypertensive } & Carvedilol & SLN & $\begin{array}{l}\text { Glyceril behenate or } \\
\text { Glyceyl palmito- } \\
\text { stearate }\end{array}$ & $\begin{array}{l}\text { ex vivo permeation and histological studies } \\
\text { in sheep nasal mucosa }\end{array}$ & 23 \\
\hline \multirow{15}{*}{$\begin{array}{l}\text { Nose-to-brain } \\
\text { delivery }\end{array}$} & \multirow{7}{*}{$\begin{array}{l}\text { Neuro- } \\
\text { degenerative } \\
\text { diseases }\end{array}$} & Anti-PD & Ropirinole & Cationic SLN & Trimyristin & $\begin{array}{l}\text { ex vivo toxicity studies on sheep nasal } \\
\text { mucosa; anti-tremor activity in albino mice } \\
\text { model }\end{array}$ & 25 \\
\hline & & \multirow{4}{*}{ Anti-AD } & Rivastigmine & SLN & $\begin{array}{l}\text { Glyceril behenate or } \\
\text { Glyceyl palmito- } \\
\text { stearate }\end{array}$ & $\begin{array}{l}\text { ex vivo permeation studies on sheep nasal } \\
\text { mucosa }\end{array}$ & 26 \\
\hline & & & Astaxanthin & SLN & Stearic acid & Biodistribution in Wistar rats & 27 \\
\hline & & & Tarenflurbil & SLN & Glyceryl monostearate & Biodistribution in Sprague Dawley rats & 28 \\
\hline & & & siRNA & Cationic SLN & $\begin{array}{l}\text { Witepsol E } 85 \text { solid } \\
\text { triglycerides }\end{array}$ & Study on Caco- 2 cell culture & 29 \\
\hline & & Anti-HD & Rosmarinic acid & SLN & Glyceryl monostearate & Functional tests in HD rat model & 30 \\
\hline & & Anti ALS & Dimethylfumarate & $\begin{array}{l}\text { SLN, cationic, } \\
\text { tween } \quad 80 \\
\text { coated }\end{array}$ & Tristearin & Bioditribution in mice & 31 \\
\hline & \multicolumn{2}{|c|}{ Antipsychotic } & Risperidone & SLN & Glyceril behenate & Biodistribution and Paw test in Balb/C mice & 32 \\
\hline & \multicolumn{2}{|c|}{ Antidepressant } & Agomelatine & SLN & Tripalmitin & Biodistribution in rats & 33,34 \\
\hline & \multicolumn{2}{|c|}{ Sedative } & Alprazolam & SLN & Glyceryl monostearate & Biodistribution in New Zealand rabbit & 35 \\
\hline & \multicolumn{2}{|l|}{ Antiepilectic } & Valproic acid & NLC & Cetyl palmitate & $\begin{array}{l}\text { Biodistribution and MES seizure test in } \\
\text { Wistar rats }\end{array}$ & 36 \\
\hline & \multicolumn{2}{|l|}{ Anti-ischemic } & Vinpocetine & $\begin{array}{l}\text { SLN bio- } \\
\text { adhesive gels }\end{array}$ & Glyceryl monostearate & $\begin{array}{l}\text { Ex-vivo bioadhesive strength, } \\
\text { histopathological and permeation studies; } \\
\text { biodistribution and pharmacokinetics }\end{array}$ & 37 \\
\hline & \multicolumn{2}{|l|}{ Antiemetic } & Ondansetron & SLN & Glyceryl monostearate & $\begin{array}{l}\text { Biodistribution in New Zealand rabbit; } \\
\text { histological studies on isolated sheep nasal } \\
\text { mucosa }\end{array}$ & 38 \\
\hline & \multicolumn{2}{|l|}{ Antitubercolar } & Streptomycin & SLN & Glyceril behenate & Biodistribution in Balb/C mice & 39 \\
\hline & \multicolumn{2}{|l|}{ Antiviral } & Efavirenz & SLN & $\begin{array}{l}\text { Tripalmitin, tristearin } \\
\text { glyceryl monostearate, } \\
\text { glyceryl behenate }\end{array}$ & Biodstribution in Wistar rats & 40 \\
\hline
\end{tabular}

Table 1: Recent overview on applications of LN in intranasal delivery

\section{iris-AperTO}

University of Turin's Institutional Research Information System and Open Access Institutional Repository 

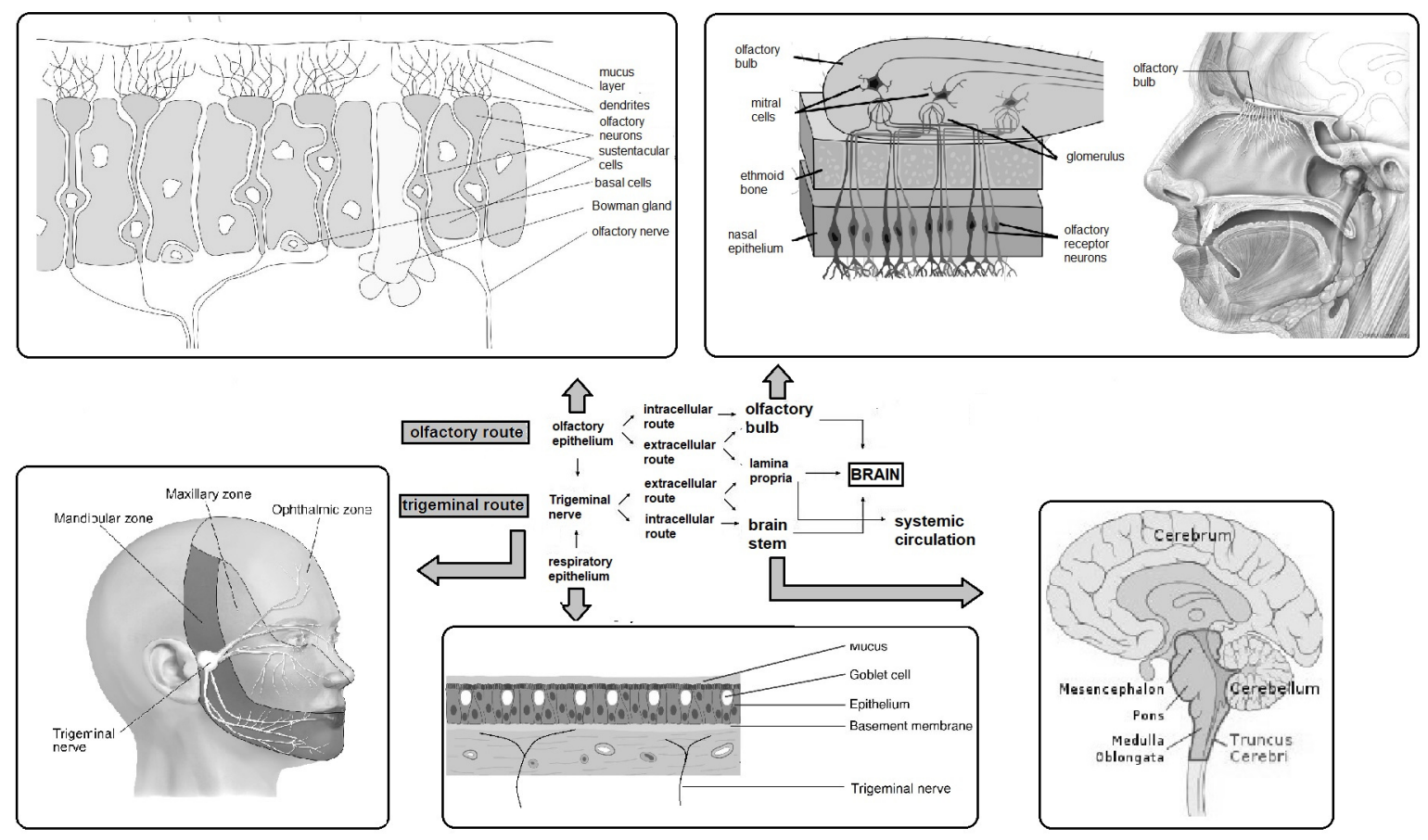

Figure 1
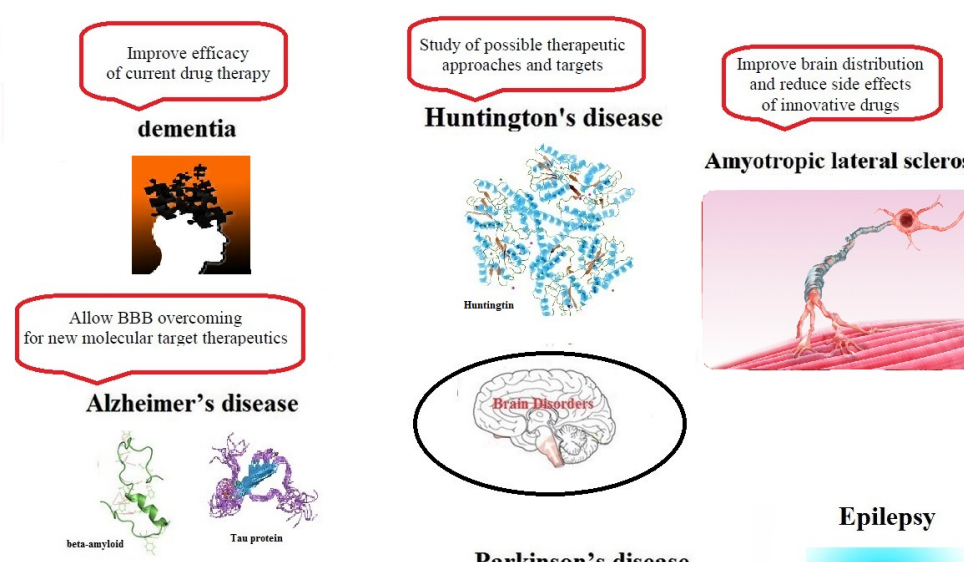

cerebrovascular diseases
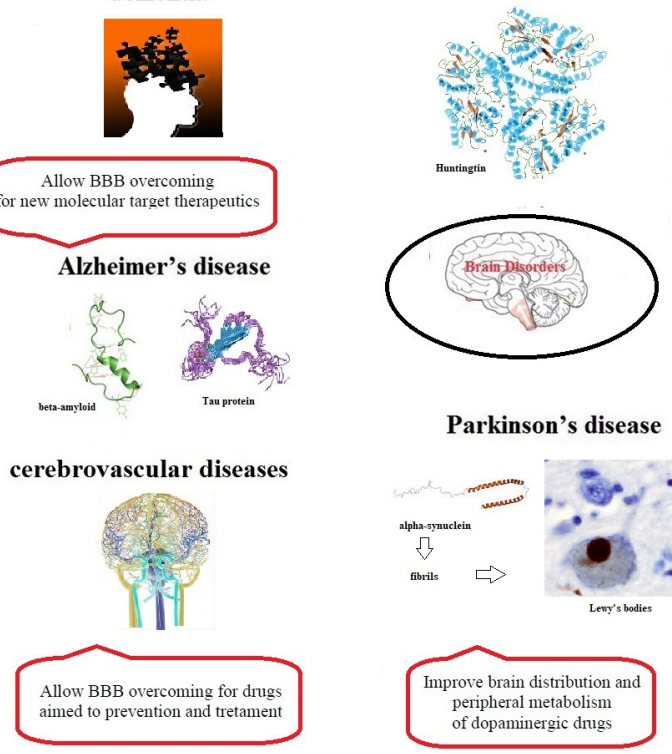

Amyotropic lateral sclerosis
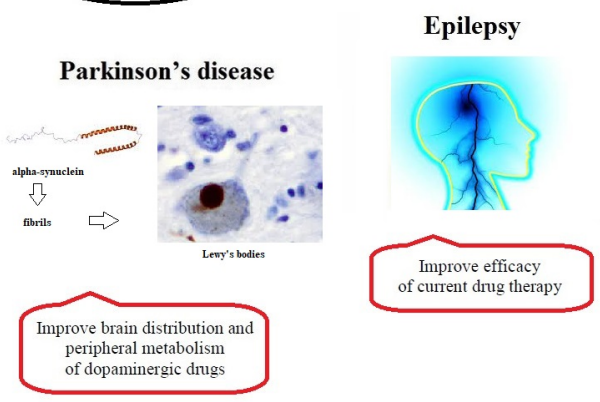

Figure 2 